\title{
Autonomous Navigation of Indoor Mobile Robot Using Global Ultrasonic System
}

\author{
Soo-Yeong Yi, Byoung-Wook Choi \\ Dept. of Electrical Engineering, Seoul National University of Technology, \\ Republic of Korea
}

\section{Introduction}

One of distinctive features of the recent intelligent autonomous robot from the conventional industrial robot is the mobility, which makes the robot overcome the limited workspace and expands it arbitrary. For autonomous navigation in workspace, a mobile robot should be able to figure out where it is and what direction it moves towards, which is called the self-localization (Singh \& Keller, 1991). The self-localization capability is the most basic requirement for mobile robots, since it is the basis of the on-line trajectory planning and control.

The trajectory error of a dead-reckoning navigation, which relies only on the internal sensor such as the odometer or the encoder, grows with time and distance. Therefore, an external sensor is necessary in order to localize the position of the robot in the workspace and to compensate it for the trajectory error. Among the several alternatives, the ultrasonic sensor is regarded as the most cost-effective external sensor and it is widely used for general purposes (Kuc \& Siegel, 1987). The methods of the self-localization using the external sensors can be classified into two groups: local methods and global methods. (1) In the local method, a mobile robot makes a local object map using the relative distance data from the environmental objects and matches the local map with a global map database. As a result, the mobile robot figures out its own position in the workspace. (2) In the global method, the mobile robot computes its position directly in the global coordinates using the distances from some reference positions in the workspace.

The local method has some advantages in that collision-avoidance motion and mapreconstruction for the transformed environment are made possible by using the distance sensors as well as the self-localization system. However, this requires massive computations in terms of the local map-making and the matching processes with the global map database. In the extreme case, the robot has to stop moving momentarily in order to obtain the necessary environmental information (Leonard \& Durrant-Whyte, 1992) (Ko et al., 1996). On the other hand, in the global method, the local map-making and the matching processes are avoidable and the self-localization is computationally efficient and fast (Leonard \& Durrante-Whyte, 1991) (Hernandez et al., 2003).

A global ultrasonic system presented in this chapter is a kind of a pseudo-lite system with a 
well-known GPS like structure (Haihang et al., 1997). For the self-localization of an indoor mobile robot, the global ultrasonic system consists of four or more ultrasonic generators fixed at reference positions in global coordinates and two receivers mounted on the mobile robot. Based on the distance measurements between the ultrasonic generators and the receivers and the appropriate data fusion algorithm for the distance data, it is possible to compute the position of the mobile robot in global coordinates.

When several ultrasonic generators are used under a system, the following problems should be taken into consideration;

(1) Cross talk between ultrasonic signals

(2) Identification of each ultrasonic signal

(3) Synchronization between each ultrasonic generator and the receiver to count the TOF(Time-Of-Flight) of ultrasonic signal

In order to solve the above problems, a small-sized RF (Radio Frequency) module is added to the ultrasonic sensors and the RF calling signal is transmitted from the ultrasonic receiver side, i.e. the mobile robot. By using this configuration, the robot is able to control triggering time and sequence of ultrasonic signal generation as well as to synchronize the ultrasonic sensors, so that to localize its own position in the global coordinates. In this chapter, we propose a global ultrasonic system and adopt the EKF (Extended Kalman Filter) algorithm designed for the self-localization. The performance of the autonomous navigation system based on the self-localization is verified through extensive experiments.

\section{A global ultrasonic system}

The overall structure of the global ultrasonic system is depicted in Fig. 1. The ultrasonic generators are fixed at known positions, $\boldsymbol{T}_{\boldsymbol{i}}=\left[x_{i}, y_{i}, z_{i}\right]^{t}, i=1, \cdots, 4$ in the work space, e.g. at each corner of the ceiling. Using the front and the rear ultrasonic sensors situated at $\boldsymbol{P}_{f}$ and $\boldsymbol{P}_{r}$, the mobile robot receives the ultrasonic signal and computes the distances by counting the TOF of the signal. It is conveniently assumed in Fig. 1 that the number of ultrasonic generators is four, which can be increased as needed in consideration of the workspace size and objects in the immediate environment. In order to avoid cross-talk between the ultrasonic signals and to synchronize the ultrasonic receivers with the generators, the RF receivers, $R X_{1} \sim R X_{4}$, and the RF transmitter, $T X$, are added to the ultrasonic generators and the ultrasonic receivers, respectively. By using the RF channel, the mobile robot sequentially activates each one of the ultrasonic generators in successive time slots. Assuming that the delivery time for the RF calling signal is negligible, the ultrasonic signal generation occurs simultaneously with the RF calling signal transmission and it is possible to synchronize the ultrasonic generators with the receivers. In Fig. $1, h_{f, 1} \sim h_{f, 4}$ denote the distance between $\boldsymbol{T}_{\boldsymbol{1}} \sim \boldsymbol{T}_{4}$ and $\boldsymbol{P}_{f}$. The distances, $h_{r, 1} \sim h_{r, 4}$, between $\boldsymbol{T}_{\boldsymbol{1}} \sim \boldsymbol{T}_{4}$ and $\boldsymbol{P}_{r}$ are omitted for brevity. The positions of the ultrasonic receivers on the robot, $\boldsymbol{P}_{\boldsymbol{f}}=\left[x_{f}, y_{f}, z_{f}\right]^{t}$ and $\boldsymbol{P}_{\boldsymbol{r}}=\left[x_{r}, y_{r}, z_{r}\right]^{t}$, with respect to the center position of the mobile robot, $\boldsymbol{P}=\left[x, y, z_{c}\right]^{t}$, can be described as follows: 


$$
\boldsymbol{P}_{f}=\left[\begin{array}{c}
x+l \cos \theta \\
y+l \sin \theta \\
z_{c}
\end{array}\right] \boldsymbol{P}_{r}=\left[\begin{array}{c}
x-l \cos \theta \\
y-l \sin \theta \\
z_{c}
\end{array}\right]
$$

where $l$ represents the distance between the center position of the mobile robot and the ultrasonic receiver, and $\theta$ denotes the heading angle of the mobile robot. It is assumed that the moving surface is flat, so that the $z$ component of the position vectors is constant as $z_{c}$ in (1).

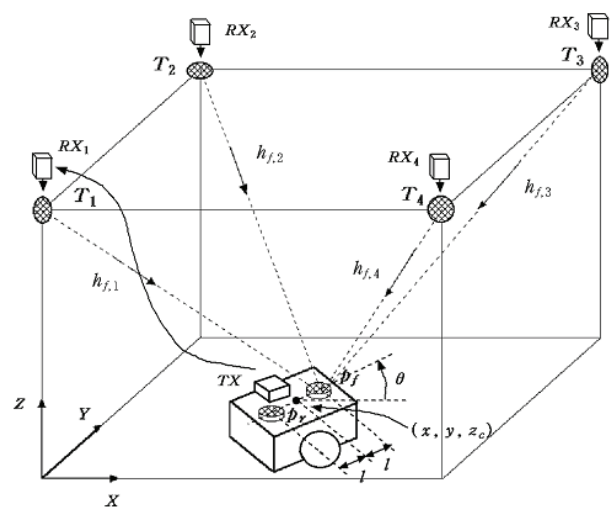

Fig. 1. A global ultrasonic system.

\section{The EKF for the self-localization and the autonomous navigation algorithm}

The position vector in the $x-y$ plane, $\boldsymbol{r}=[x, y]^{t}$, together with the heading angle, $\theta$, of a mobile robot having differential wheels, follows the state equation (2) in the discrete-time domain (Fox et al., 1997):

$$
\left[\begin{array}{c}
x_{k+1} \\
y_{k+1}
\end{array}\right]=\left\{\begin{array}{c}
{\left[\begin{array}{c}
x_{k}+T v_{k} \cos \theta_{k} \\
y_{k}+T v_{k} \sin \theta_{k}
\end{array}\right]} \\
{\left[\begin{array}{c}
x_{k}+\rho_{k} \cos \theta_{k} \sin \left(T \omega_{k}\right) \\
-\rho_{k} \sin \theta_{k}\left(1-\cos \left(T \omega_{k}\right)\right) \\
x_{k}+\rho_{k} \cos \theta_{k} \sin \left(T \omega_{k}\right) \\
+\rho_{k} \sin \theta_{k}\left(1-\cos \left(T \omega_{k}\right)\right)
\end{array}\right] \quad \text { if } \omega_{k} \neq 0} \\
\theta_{k+1}=\theta_{k}+T \omega_{k}
\end{array}\right.
$$

where the subscript $k$ is the time index, $T$, denotes the sampling interval, $v_{k}$ and $\omega_{k}$ are the linear and the angular velocities of the robot respectively, and $\rho_{k}=\frac{v_{k}}{\omega_{k}}$ represents the radius of rotation. The position vector and the heading angle of the mobile robot are augmented so as to become $\boldsymbol{P}=[x, y, \theta]^{t}$, which is referred to as the robot posture. The bold and normal symbols represent the vector and the scalar variables, respectively. 
As a consequence of (1) and (2), the state equation for the ultrasonic receivers on the robot can be described as follows:

$$
\begin{aligned}
& \boldsymbol{r}_{f, k+1}=\boldsymbol{f}_{f}\left(\boldsymbol{r}_{f, k}, \boldsymbol{u}_{k}, \boldsymbol{q}_{k}\right)
\end{aligned}
$$

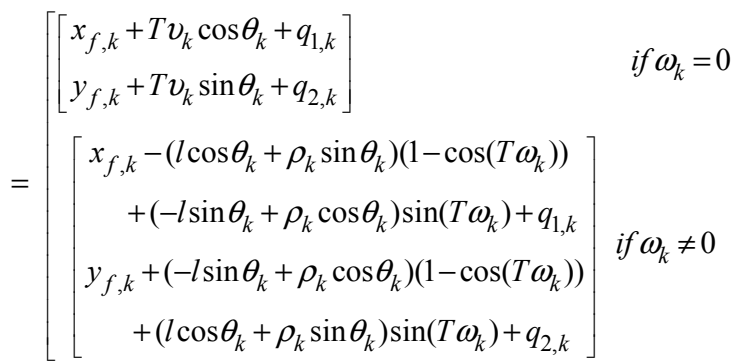

$$
\begin{aligned}
& \boldsymbol{r}_{r, k+1}=\boldsymbol{f}_{r}\left(\boldsymbol{r}_{r, k}, \boldsymbol{u}_{k}, \boldsymbol{q}_{k}\right) \\
& =\left[\begin{array}{c}
{\left[\begin{array}{l}
x_{r, k}+T v_{k} \cos \theta_{k}+q_{1, k} \\
y_{r, k}+T v_{k} \sin \theta_{k}+q_{2, k}
\end{array}\right]} \\
{\left[\begin{array}{r}
x_{r, k}-\left(l \cos \theta_{k}+\rho_{k} \sin \theta_{k}\right)\left(1-\cos \left(T \omega_{k}\right)\right) \\
+\left(-l \sin \theta_{k}+\rho_{k} \cos \theta_{k}\right) \sin \left(T \omega_{k}\right)+q_{1, k} \\
y_{r, k}+\left(-l \sin \theta_{k}+\rho_{k} \cos \theta_{k}\right)\left(1-\cos \left(T \omega_{k}\right)\right) \\
+\left(l \cos \theta_{k}+\rho_{k} \sin \theta_{k}\right) \sin \left(T \omega_{k}\right)+q_{2, k}
\end{array}\right]}
\end{array}\right] \text { if } \omega_{k} \neq 0
\end{aligned}
$$

where $\boldsymbol{r}_{\boldsymbol{f}}=\left[x_{f}, y_{f}\right]^{t}$ and $\boldsymbol{r}_{\boldsymbol{r}}=\left[x_{r}, y_{r}\right]^{t}$ represent the positions of the front and rear ultrasonic receivers respectively and $\boldsymbol{q}_{\boldsymbol{k}}=\left[q_{1, k}, q_{2, k}\right]^{t}$ is the Gaussian random noise with zero mean and $\boldsymbol{Q}$ variance. The measurement equation at the ultrasonic receivers can be modeled as follows:

$$
\begin{aligned}
z_{f, k} & =h_{f, i}\left(\boldsymbol{r}_{f, k}, v_{k}\right) \\
= & \left\{\left(x_{f, k}-x_{i}\right)^{2}+\left(y_{f, k}-y_{i}\right)^{2}+\left(z_{c}-z_{i}\right)^{2}\right\}^{1 / 2}+v_{k} \\
z_{r, k} & =h_{r, i}\left(\boldsymbol{r}_{r, k}, v_{k}\right) \\
= & \left\{\left(x_{r, k}-x_{i}\right)^{2}+\left(y_{r, k}-y_{i}\right)^{2}+\left(z_{c}-z_{i}\right)^{2}\right\}^{1 / 2}+v_{k}
\end{aligned}
$$

where the measurement noise, $v_{k}$, is assumed to be Gaussian with zero mean and $G$ variance, and the subscript, $i$, denotes one of the ultrasonic generators, $\boldsymbol{T}_{\boldsymbol{1}} \sim \boldsymbol{T}_{\boldsymbol{4}}$, which is called by the mobile robot at time $k$.

From the state Eq. (3-1) and the measurement Eq. (4-1), it is possible to get the following set of equations constituting the EKF estimation for the front ultrasonic receiver position:

$$
\begin{aligned}
\hat{\boldsymbol{r}}_{f, k+1}^{-} & =\boldsymbol{f}_{f}\left(\hat{\boldsymbol{r}}_{f, k}, \boldsymbol{u}_{k}, \mathbf{0}\right) \\
\boldsymbol{V}_{f, k+1}^{-} & =\boldsymbol{A}_{f, k} \boldsymbol{V}_{f, k} \boldsymbol{A}_{f, k}^{t}+\boldsymbol{Q}
\end{aligned}
$$




$$
\begin{aligned}
\boldsymbol{K}_{f, k} & =\boldsymbol{V}_{f, k}^{-} \boldsymbol{H}_{f, k}{ }^{t}\left(\boldsymbol{H}_{f, k} \boldsymbol{V}_{f, k}^{-} \boldsymbol{H}_{f, k}{ }^{t}+G\right)^{-1} \\
\boldsymbol{V}_{f, k} & =\left(\boldsymbol{I}-\boldsymbol{K}_{f, k} \boldsymbol{H}_{f, k}\right) \boldsymbol{V}_{f, k}^{-} \\
\hat{\boldsymbol{r}}_{f, k} & =\hat{\boldsymbol{r}}_{f, k}^{-}+\boldsymbol{K}_{f, k}\left(z_{f, k}-h_{f, i}\left(\hat{\boldsymbol{r}}_{f, k}^{-}, \mathbf{0}\right)\right)
\end{aligned}
$$

where $\boldsymbol{K}_{f, k}$ is the kalman filter gain, $\hat{\boldsymbol{r}}_{f, k}^{-}$and $\hat{\boldsymbol{r}}_{f, k}$ represents the a priori and a posteriori estimations for $\boldsymbol{r}_{f, k^{\prime}}$, respectively, and $\boldsymbol{V}_{f, k}{ }^{-}$and $\boldsymbol{V}_{f, k}$ represent the a priori and a posteriori error covariance matrices, respectively, as defined in (7).

$$
\begin{aligned}
\boldsymbol{V}_{f, k}^{-} & =\boldsymbol{E}\left[\left(\boldsymbol{r}_{f, k}-\hat{\boldsymbol{r}}_{f, k}^{-}\right)\left(\boldsymbol{r}_{f, k}-\hat{\boldsymbol{r}}_{f, k}^{-}\right)^{t}\right] \\
\boldsymbol{V}_{f, k} & =\boldsymbol{E}\left[\left(\boldsymbol{r}_{f, k}-\hat{\boldsymbol{r}}_{f, k}\right)\left(\boldsymbol{r}_{f, k}-\hat{\boldsymbol{r}}_{f, k}\right)^{t}\right]
\end{aligned}
$$

where $\boldsymbol{E}(\cdot)$ denotes the expectation of the corresponding random variables. The Jacobian matrices, $\boldsymbol{A}_{f, k}$ and $\boldsymbol{H}_{f, k}$, in (6) are given as follows:

$$
\begin{aligned}
\boldsymbol{A}_{f, k} & =\frac{\partial \boldsymbol{f}_{f}}{\partial \boldsymbol{r}_{f, k}}\left(\hat{\boldsymbol{r}}_{f, k}, \boldsymbol{u}_{k}, \mathbf{0}\right) \\
& =\left[\begin{array}{ll}
1 & 0 \\
0 & 1
\end{array}\right] \\
\boldsymbol{H}_{f, k} & =\frac{\partial h_{f, i}}{\partial \boldsymbol{r}_{f, k}}\left(\hat{\boldsymbol{r}}_{f, k}, \mathbf{0}\right) \\
& =\left[\begin{array}{ll}
\frac{x_{f, k}-x_{i}}{D_{f, i}} & \frac{y_{f, k}-y_{i}}{D_{f, i}}
\end{array}\right]
\end{aligned}
$$

where $D_{f, i}$ is defined by the following Eq. (10).

$$
D_{f, i}=\left\{\left(x_{f, k}-x_{i}\right)^{2}+\left(y_{f, k}-y_{i}\right)^{2}+\left(z_{c}-z_{i}\right)^{2}\right\}^{1 / 2}
$$

The EKF estimation, $\hat{\boldsymbol{r}}_{r, k}$, for the rear ultrasonic receiver position is similar and omitted here for the sake of brevity.

From $\hat{\boldsymbol{r}}_{f, k}$ and $\hat{\boldsymbol{r}}_{r, k}$, the posture estimation for the mobile robot can be described as follows:

$$
\begin{aligned}
& \hat{x}_{k}=\frac{\hat{x}_{f, k}+\hat{x}_{r, k}}{2} \\
& \hat{y}_{k}=\frac{\hat{y}_{f, k}+\hat{y}_{r, k}}{2} \\
& \hat{\theta}_{k}=\tan ^{-1} \frac{\hat{y}_{f, k}-\hat{y}_{r, k}}{\hat{x}_{f, k}-\hat{x}_{r, k}}
\end{aligned}
$$

Assuming that the estimation error covariances for positions of the front and the rear ultrasonic receiver are the same, the error covariances of the posture estimation are given in (12) as shown in Fig. 2. 


$$
\begin{aligned}
\boldsymbol{V}_{p, k} & =\boldsymbol{E}\left[\left(\boldsymbol{r}-\hat{\boldsymbol{r}}_{k}\right)\left(\boldsymbol{r}-\hat{\boldsymbol{r}}_{k}\right)^{2}\right] \\
& =\boldsymbol{V}_{f, k}\left(=\boldsymbol{V}_{r, k}\right) \\
\boldsymbol{V}_{\theta, k} & =E\left[\left(\theta-\theta_{k}\right)^{2}\right] \\
& \approx \tan ^{-1} \frac{\boldsymbol{V}_{f, k}}{l}
\end{aligned}
$$

Eq. (12) implies that the estimation for heading angle becomes more accurate according to the distance between the two ultrasonic receivers. Based on the self-localization given in (11), a simple control input, $v_{k}$ and $\omega_{k}$, to drive the mobile robot toward the given goal position, can be written as (13).

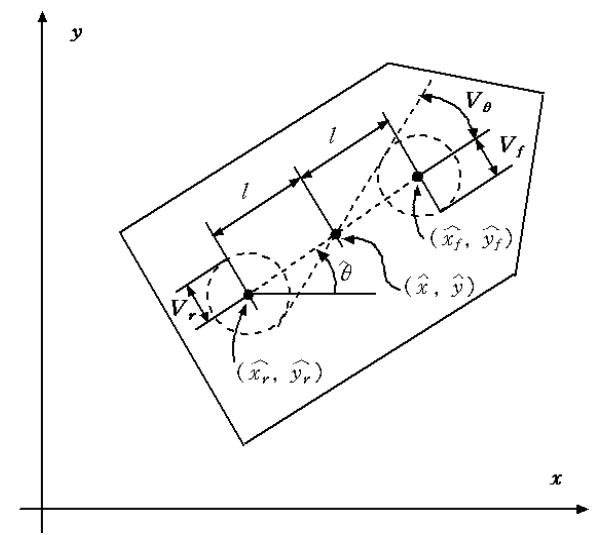

Fig. 2. Error covariances of the posture estimation.

$$
\begin{aligned}
& v_{k}=c \\
& \omega_{k}=k_{\theta}\left(\theta_{d, k}-\theta_{k}\right), \quad \theta_{d, k}=\tan ^{-1} \frac{y_{g}-\hat{y}_{k}}{x_{g}-\hat{x}_{k}}
\end{aligned}
$$

where $c$ and $k_{\theta}$ are positive constants. The mobile robot adjusts its heading angle toward the intended position and moves with the constant velocity as depicted in Fig. 3.

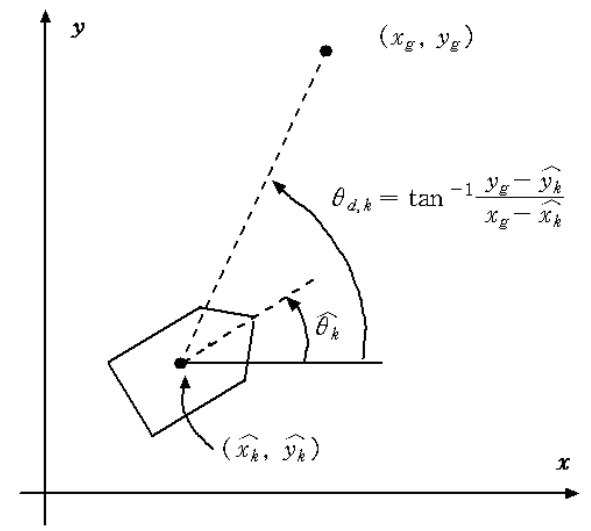

Fig. 3. Navigation control. 


\section{Experiments and Discussions}

In order to verify the performance of the EKF based self-localization and autonomous navigation system using the global ultrasonic system, a simple experimental set-up was established as shown in Fig. 4, which has dimension of 1,500 $\mathrm{mm}$ and 1,500 $\mathrm{mm}$ in width and length respectively and 2,500 $\mathrm{mm}$ in height. The ultrasonic generators installed with the RF receivers are fixed near the four corners of the ceiling and their positions are described in (14).

Fig. 4. Experimental set-up.

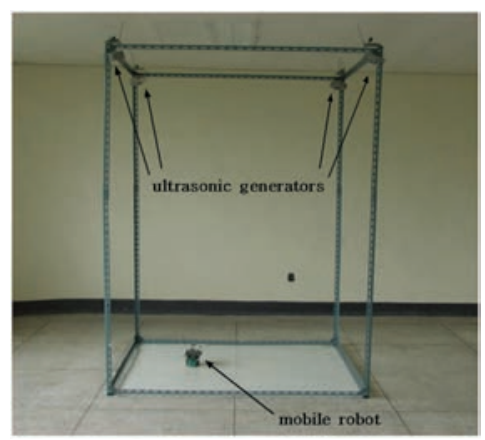

$$
\begin{aligned}
& \boldsymbol{T}_{1}=[10.0,10.0,2360.0]^{t} \\
& \boldsymbol{T}_{2}=[1427.0,5.0,2370.0]^{t} \\
& \boldsymbol{T}_{3}=[1423.0,1445.0,2357.0]^{t} \\
& \boldsymbol{T}_{4}=[0.0,1380.0,2370.0]^{t}
\end{aligned}
$$

At first, a preliminary experiment was carried out for the ultrasonic calibration and the result is presented in Fig. 5.

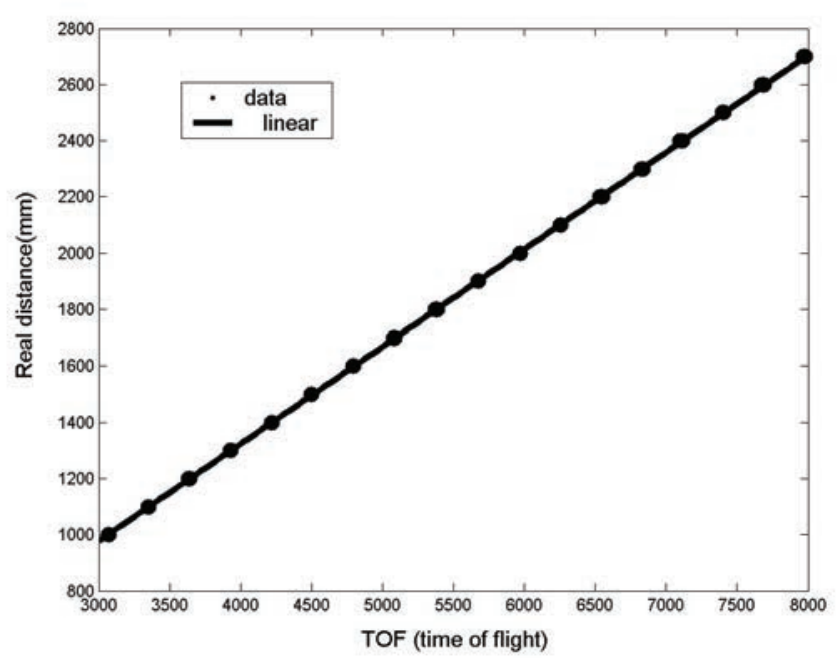

Fig. 5. Real distance with respect to ultrasonic TOF. 
The linear equation relating the ultrasonic TOF to the real distance is given in (15), as obtained from the least-square method, and the variance of the measurement noise is specified as (16).

$$
\begin{gathered}
D=0.34533 \cdot T-57.224 \\
G=1.8
\end{gathered}
$$

where $D(\mathrm{~mm})$ represents the real distance between the ultrasonic generator and the receiver and $T(\mu \mathrm{sec})$ is the ultrasonic TOF.

Fig. 6 shows the results of the self-localization experiment, in which the robot is moved manually from the initial posture, $(x, y, \theta)=(600,600,0)$ to the goal posture, $(900,900, \pi / 2)$ at $45 \mathrm{sec}$. The initial value of the posture estimation is set arbitrarily as $(650,650,0)$. The distance and the heading angle are described by $m m$ and rad., respectively. As shown in Fig. 6, the position errors in the $x$ and $y$ axes are less than $25 \mathrm{~mm}$ in the steady-state. Since the distance between the center position of the robot and the ultrasonic receiver is designed as $l=75 \mathrm{~mm}$, the estimation error of the heading angle in (12) becomes $\tan ^{-1}(25 / 75) \approx 0.32 \mathrm{rad}$. as shown in Fig. 6 (c).

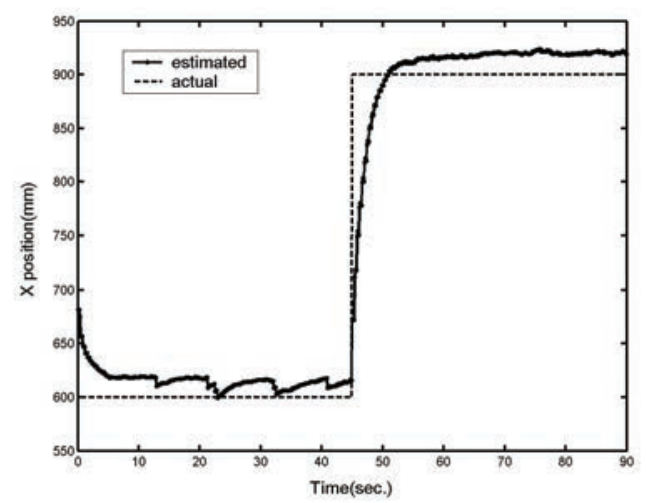

(a) Position estimation in $x$ axis.

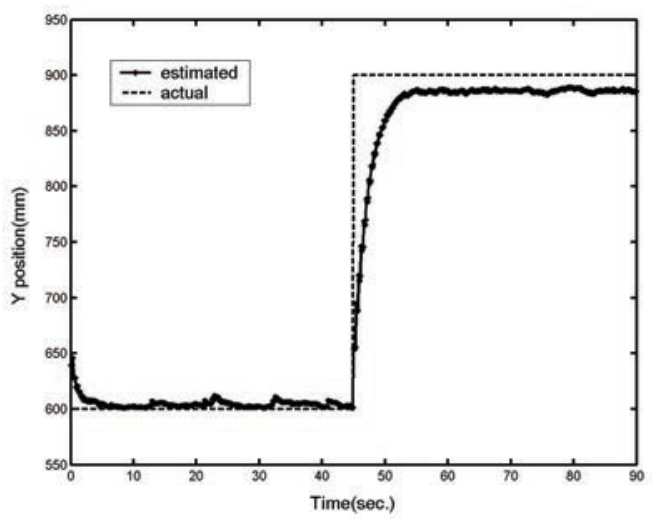

(b) Position estimation in $y$ axis. 


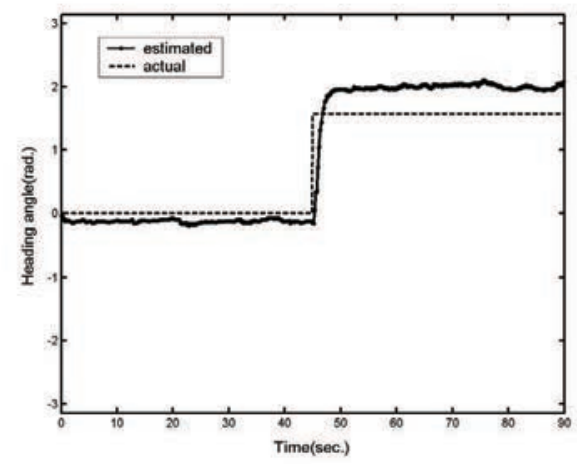

(c) Estimation for heading ${ }_{\theta}$ angle.

Fig. 6. The self-localization of the mobile robot.

The autonomous navigation system using the global ultrasonic system is compared to the dead-reckoning navigation system on the straight line connecting the initial posture, $(650,650, \pi / 4)$, and the goal posture, $(900,900, \pi / 4)$, in the workspace.

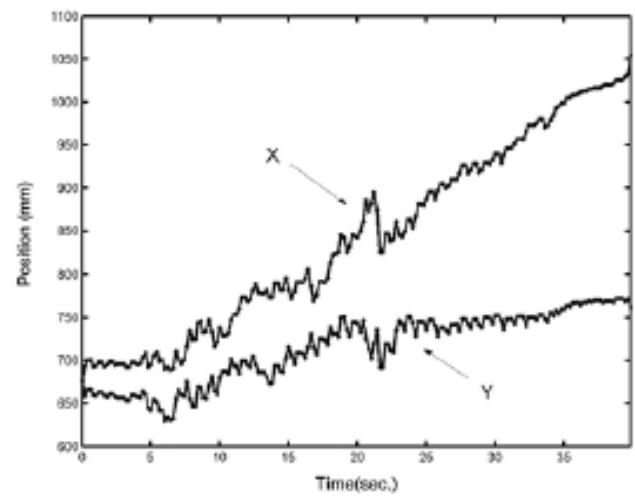

(a) Position in $x$ and $y$ axis

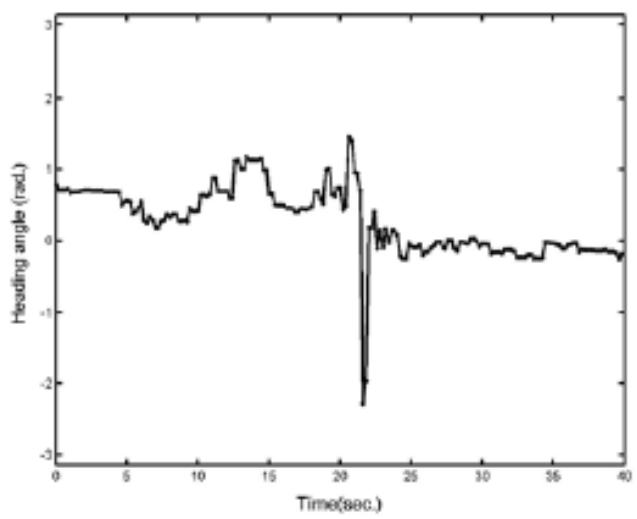

(b) Heading angle 


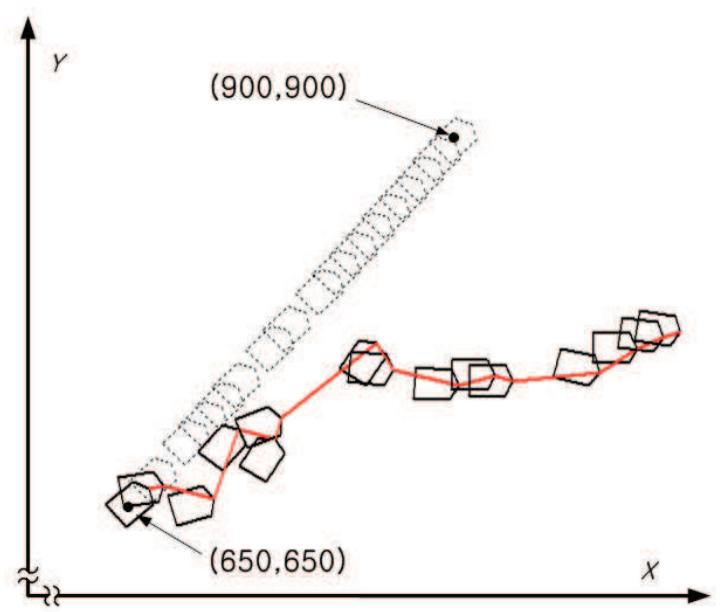

(c) Trajectory in $x-y$ plane

Fig. 7. The dead-reckoning navigation.

Fig. 7 shows the results in the case of the dead-reckoning navigation, in which the mobile robot cannot reach its goal posture, due to the uncertainties in the state equation. In Fig. 7 (c), the dotted polygons represent the desired postures of the mobile robot with respect to time. The results of the autonomous navigation system based on the self-localization using the global ultrasonic system are presented in Fig. 8 for the same initial and goal postures. As shown in this figure, the mobile robot reaches the goal posture, overcoming the uncertainties in the state equation, and the heading angle at the final position is around $\frac{\pi}{4}$ as desired. It should be noted that the posture data in Figs. 7 and 8 are obtained by using the global ultrasonic system also, thus these values may be different from the actual postures to some degree.

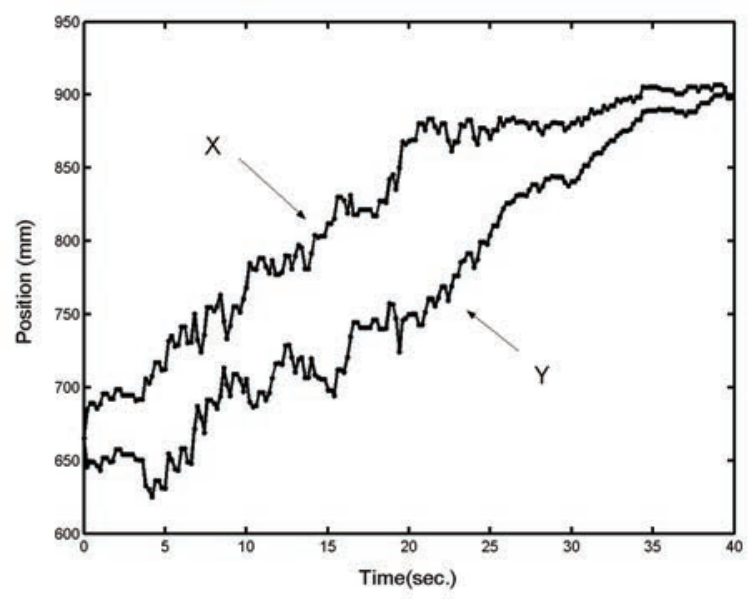

(a) Position in $x$ and $y$ axis 


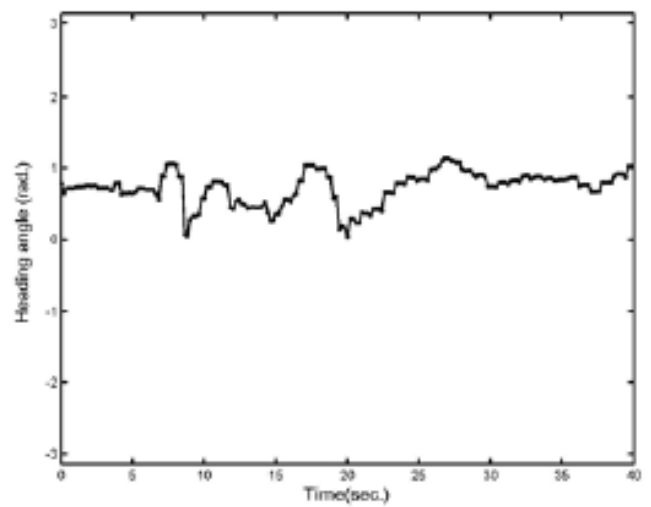

(b) Heading angle

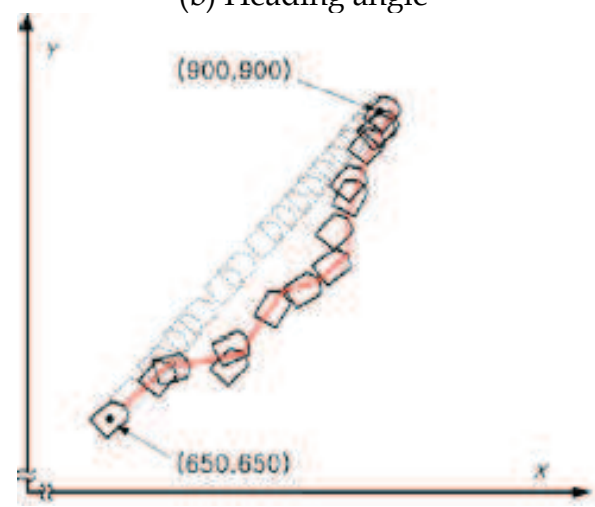

(c) Trajectory in $x-y$ plane

Fig. 8. Navigation with global ultrasonic system.

The size of the ultrasonic region in the work space is dependant on the beam-width of the ultrasonic generator. In the case of a general ultrasonic ranging system, in which both the signal generator and the receiver are lumped together, an ultrasonic generator with a narrow beam-width is preferable in order to avoid the ambiguity and to enhance the measurement accuracy. On the other hand, the proposed global ultrasonic system, which has a distributed signal generator, requires the use of a wide beam-width generator, in order to expand the ultrasonic region in the work space.

\section{Conclusions}

In this chapter, the global ultrasonic system with an EKF algorithm is presented for the selflocalization of an indoor mobile robot. Also, the performance of the autonomous navigation based on the self-localization system is thus verified through various experiments. The global ultrasonic system consists of four or more ultrasonic generators fixed at known positions in the workspace, two receivers mounted on the mobile robot, and RF modules added to the ultrasonic sensors. By controlling the ultrasonic signal generation through the $\mathrm{RF}$ channel, the robot can synchronize and measure the distance between the ultrasonic generators and receivers, thereby estimating its own position and heading angle. It is shown 
through experiments that the estimation errors are less than $25 \mathrm{~mm}$ in terms of the position and less than $0.32 \mathrm{rad}$. in terms of the heading angle. Since the estimation error of the heading angle is dependant on the distance between the two ultrasonic receivers on the robot, it is possible to obtain a more accurate estimation for the heading angle by increasing this distance.

The global ultrasonic system has the following salient features: (1) simple and efficient state estimation since the process of local map-making and matching with the global map database is avoidable due to the GPS-like nature of the system, (2) active cuing of the ultrasonic generation time and sequence through the RF channel, and (3) robustness against signal noise, since the ultrasonic receiver on the mobile robot processes the signal received directly from the generator, instead of through an indirect reflected signal.

In this chapter, it is assumed that an ideal environment exists without any objects in the workspace. Environmental objects may result in an area of relative obscurity, which the ultrasonic signals cannot reach. It is possible to overcome the problems associated with environments containing obstacles by increasing the number of ultrasonic generators in the work space as needed. This enhancement is currently being studied.

\section{References}

Fox, D.; Burgard, W. \& Thrun, S. (1997). The dynamic window approach to collision avoidance, IEEE Robotics and Automation Magazine, Vol.4, No.1, March, pp.23-33, ISSN:1070-9932

Haihang, S; Muhe, G. \& Kezhong, H. (1997). An integrated GPS/CEPS position estimation system for outdoor mobile robot, Proceedings of IEEE International Conference on Intelligent Processing Systems, Beijing, China, October, pp.28-31

Hernandez, S.; Torres, J.M, Morales, C.A. \& Acosta, L. (2003). A new low cost system for autonomous robot heading and position localization in a closed area, Autonomous Robots, Vol. 15, pp. 99-110, ISSN:0929-5593

Kleeman, L. (1992). Optimal Estimation of Position and Heading for Mobile Robots Using Ultrasonic Beacons and Dead-reckoning, Proceedings of IEEE Conference on Robotics and Automations, Nice, France, May, pp.2582-2587

Ko, J.; Kim, W. \& Chung, M. (1996). A Method of Acoustic Landmark Extraction for Mobile Robot Navigation, IEEE Transaction on Robotics and Automation, Vol.12, No.6, pp.478-485, ISSN:1552-3098

Leonard, J.; Durrant-Whyte, H. (1991). Mobile Robot Localization by Tracking Geometric Beacons, IEEE Transaction on Robotics and Automation, Vol.7, No.3, pp.376-382, ISSN:1552-3098

Leonard, J. \& Durrant-Whyte, H. (1992). Directed sonar sensing for mobile robot navigation, Kluwer Academic Publishers, ISBN:0792392426

R. Kuc \& Siegel, M.W. (1987). Physically based simulation model for acoustic sensor robot navigation. IEEE Transaction on Pattern Analysis and. Machine Intelligence, Vol.9, No.6, pp.766-777, ISSN:0162-8828

Singh, S. \& Keller, P. (1991). Obstacle detection for high speed autonomous navigation. Proceedings of IEEE International Conference on Robotics and Automation, pp.2798-2805 


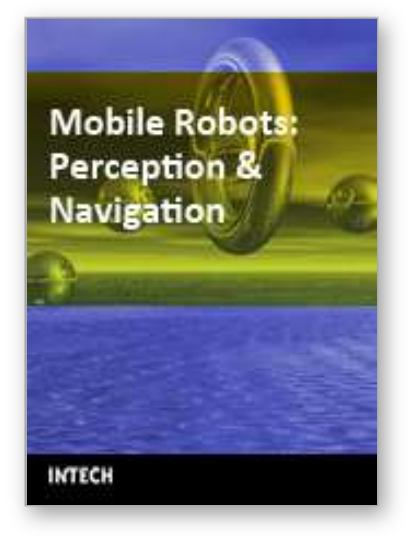

\author{
Mobile Robots: Perception \& Navigation \\ Edited by Sascha Kolski
}

ISBN 3-86611-283-1

Hard cover, 704 pages

Publisher Pro Literatur Verlag, Germany / ARS, Austria

Published online 01, February, 2007

Published in print edition February, 2007

Today robots navigate autonomously in office environments as well as outdoors. They show their ability to beside mechanical and electronic barriers in building mobile platforms, perceiving the environment and deciding on how to act in a given situation are crucial problems. In this book we focused on these two areas of mobile robotics, Perception and Navigation. This book gives a wide overview over different navigation techniques describing both navigation techniques dealing with local and control aspects of navigation as well es those handling global navigation aspects of a single robot and even for a group of robots.

\title{
How to reference
}

In order to correctly reference this scholarly work, feel free to copy and paste the following:

Soo-Yeong Yi and Byoung-Wook Choi (2007). Autonomous Navigation of Indoor Mobile Robot Using Global Ultrasonic System, Mobile Robots: Perception \& Navigation, Sascha Kolski (Ed.), ISBN: 3-86611-283-1, InTech, Available from:

http://www.intechopen.com/books/mobile_robots_perception_navigation/autonomous_navigation_of_indoor_m obile_robot_using_global_ultrasonic_system

\section{INTECH}

open science | open minds

\section{InTech Europe}

University Campus STeP Ri

Slavka Krautzeka 83/A

51000 Rijeka, Croatia

Phone: +385 (51) 770447

Fax: +385 (51) 686166

www.intechopen.com

\section{InTech China}

Unit 405, Office Block, Hotel Equatorial Shanghai

No.65, Yan An Road (West), Shanghai, 200040, China 中国上海市延安西路65号上海国际贵都大饭店办公楼 405 单元

Phone: $+86-21-62489820$

Fax: $+86-21-62489821$ 
(C) 2007 The Author(s). Licensee IntechOpen. This chapter is distributed under the terms of the Creative Commons Attribution-NonCommercialShareAlike-3.0 License, which permits use, distribution and reproduction for non-commercial purposes, provided the original is properly cited and derivative works building on this content are distributed under the same license. 